\title{
Traumatismo torácico: Análisis de hospitalizaciones según grupo etario
}

\author{
Roberto González L. ${ }^{1,2}$, Sebastián Barra M. ${ }^{3}$, Alejandra Riquelme U. ${ }^{4}$, \\ Rodrigo Reyes M. ${ }^{1,2}$, Felipe Alarcón O. ${ }^{3}$, Enrique Seguel S. ${ }^{1,2}$, Aleck Stockins L. ${ }^{1,2}$, \\ Andrés Jadue T. ${ }^{1}$, Andrés Schaub C. ${ }^{3}$, Diego Saldivia Z. ${ }^{3}$ y Emilio Alarcón C. ${ }^{1,2}$
}

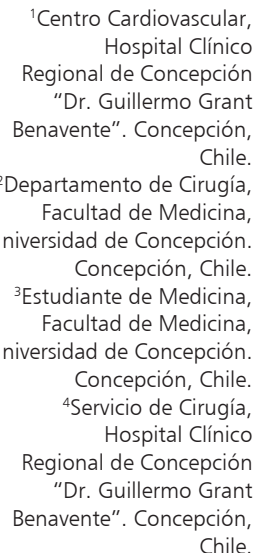

Recibido 2020-05-22 y aceptado 2020-06-30

Esta comunicación corresponde a uno de los análisis de la base de datos prospectiva de hospitalizaciones por traumatismo torácico iniciada el año 1981 en Concepción, Chile.

Correspondencia a: Dr. Roberto González L. rgonzalezlagos@udec.cl

\section{Thoracic trauma: analysis of hospitalizations among different age-groups}

Background: Trauma is the leading death cause among adolescents and the fifth in the elderly. Thoracic trauma (TT) has particular characteristics in different age-groups. Aim: To analyze clinical characteristics, trauma severity indices (TSI) and mortality in patients hospitalized for TT among different age-groups. Materials and Method: Descriptive study of patients hospitalized for TT between January-1981 and December-2018. Prospective TT surgical operation notes and data base were reviewed. Age-groups were determined according to the World Health Organization (Adolescent: 10-19 years; Young Adult: 20-44 years; Mature Adult: 45-59 years; Elderly > 60 years). TT classification, mechanism, agent, treatment, TSI, Trauma Injury Severity Score (TRISS) and mortality were compared among age-groups. SPSS25 ${ }^{\circledR}$ with chi-square test and ANOVA were used, considering $p<0.05$ significant. Results: Total 4.297 TT. Adolescent age-group: 608 (14.1\%); Young Adult: 2,544 (59.2\%); Mature Adult: 601 (14.0\%); Elderly: $544(12.7 \%)$. Was observed a progressive and significant decrease of penetrating TT, aggression-caused and bladed weapon-caused TT from Adolescent to Elderly. In Young Adult the invasive treatment predominant: surgery 541 (21.2\%), whereas in Mature Adult TT with associated injuries $215(35.8 \%), p<0.05$. Differences in TRISS and mortality $1.6 \% ; 2.5 \% ; 3.4 \% ; 5.0 \%(\mathrm{p}<0.05)$ were observed among age-groups, respectively. Conclusions: There are statistical significant differences in clinical characteristics, TSI and mortality when comparing TT by age group. Age is an important factor determining the outcome in TT hospitalized patients.

Key words: wounds and injuries; thoracic injuries; age groups; trauma severity indices; thoracic surgery.

\section{Resumen}

Introducción: El traumatismo es la primera causa de muerte en adolescentes y la quinta en el adulto mayor. El traumatismo torácico (TT) posee características particulares en diferentes grupos de edad. Objetivos: Analizar características clínicas, índice de gravedad de traumatismo (IGT) y mortalidad en hospitalizados por TT según grupo etario. Materiales y Método: Estudio descriptivo de hospitalizaciones por TT, período enero de 1981 a diciembre de 2018. Revisión de protocolos prospectivos de TT y base de datos. Se definió grupo etario según Organización Mundial de la Salud (Adolescente: 10-19 años; Adulto Joven: 20-44 años; Adulto Maduro 45-59 años; Adulto Mayor: $\geq 60$ años). Se comparó clasificación, mecanismo, agente, tratamiento, IGT, Trauma Injury Severity Score (TRISS) y mortalidad del TT según grupo etario. Se utilizó SPSS25® con pruebas chi-cuadrado y ANOVA, considerando significativo $\mathrm{p}<$ 0,05. Resultados: Total 4.297 TT. Grupo etario Adolescente: 608 (14,1\%); Adulto Joven: 2.544 (59,2\%); Adulto Maduro: 601 (14,0\%); Adulto Mayor: 544 (12,7\%). Se observó disminución progresiva y significativa en TT penetrante, por agresión y del TT por arma blanca desde grupo etario Adolescente hasta Adulto Mayor. En Adulto Joven predominó tratamiento invasivo: cirugía 541 (21,2\%) y en Adulto Maduro el TT con lesiones asociadas $215(35,8 \%), p<0,05$. Según grupo etario, se observaron diferencias significativas en TRISS y en mortalidad. La mortalidad fue $1,6 \% ; 2,5 \% ; 3,3 \% ; 5,0 \%$, según grupo etario respectivamente $(p<0,05)$. Conclusiones: Existen diferencias estadísticamente significativas en las características clínicas, IGT y mortalidad del TT al comparar distintos grupos etarios. La edad es uno de los factores que determina el pronóstico de pacientes hospitalizados por TT.

Palabras clave: heridas y traumatismos; traumatismos torácicos; grupos de edad; puntaje de gravedad del traumatismo; cirugía torácica. 


\section{Introducción}

Los traumatismos son una de las principales causas de hospitalización y mortalidad en todos los grupos etarios a nivel mundial. Se estima que el traumatismo es la primera causa de muerte en adolescentes y la quinta en adultos mayores, siendo entre el $25 \%-45 \%$ de ellas atribuibles a un traumatismo torácico $(\mathrm{TT})^{1-4}$.

En nuestro país los traumatismos corresponden a un problema de salud pública vigente: aproximadamente un quinto del total de las defunciones son producidas a consecuencia de traumatismos, envenenamientos y otras causas externas ${ }^{5}$, provocando alrededor de un tercio del total de años de vida potencialmente perdidos en $\mathrm{Chile}^{6}$.

Además, los traumatismos representan la cuarta causa de egresos hospitalarios, siendo el TT el diagnóstico principal en el 5\%-10\% de ellos. Las hospitalizaciones por TT son más frecuentes en hombres que en mujeres con relación 7:1 y la mayoría se concentra en población laboralmente activa ${ }^{7}$.

Se describe que el TT posee diferentes características entre distintos rangos etarios, lo que está determinado principalmente por las variables sociodemográficas de la serie estudiada. Así, en series donde predomina el TT contuso, existe mayor proporción de adultos mayores con lesiones graves, lo que determina una mayor mortalidad principalmente a expensas de accidentes de tránsito ${ }^{3,8}$. Por otro lado, en series donde predomina el TT penetrante, se ha descrito que tanto el mecanismo del traumatismo, las lesiones asociadas o la necesidad de intervención quirúrgica pueden ser similares entre adolescentes y adultos ${ }^{9,10}$.

Estudios demuestran que la tolerancia de los tejidos humanos al traumatismo disminuye con la edad ${ }^{11}$. Particularmente en el TT, frente a traumatismos de similar energía, las personas jóvenes sufren menos complicaciones tanto intra como extratorácicas, teniendo menor necesidad de cuidados intensivos y estadía hospitalaria versus personas ancianas, en las que la morbilidad y mortalidad asociada al traumatismo suele ser significativamente mayor ${ }^{11,12}$.

En una publicación reciente de nuestro grupo se demostró un aumento de las hospitalizaciones por TT, asociado a un aumento progresivo de las hospitalizaciones en adultos mayores, los cuales poseen cualidades particulares que los diferencian de la población general ${ }^{13,14}$. Sin embargo, y aunque las características del TT han sido bien documentadas en grupos seleccionados ${ }^{15-18}$, existen escasas publicaciones en nuestro medio que describan y comparen al TT entre diferentes rangos de edad.
Nuestros objetivos son analizar las principales características clínicas, morbilidad y mortalidad de pacientes hospitalizados por TT según grupo etario.

\section{Materiales y Método}

Estudio descriptivo transversal de pacientes hospitalizados por TT en el Hospital Clínico Regional "Dr. Guillermo Grant Benavente" de Concepción, Chile, tratados por el equipo de Cirugía Cardiotorácica entre enero de 1981 y diciembre de 2018.

Se dividió la serie en grupos etarios de acuerdo a los criterios utilizados por la Organización Mundial de la Salud ${ }^{19,20}$ : Adolescente 10-19 años; Adultos 20-59 años y Adulto Mayor 60 o más años. Adicionalmente, y para efectos del análisis, se subdividió el grupo de adultos en: Adulto Joven 20-44 años y Adulto Maduro 45-59 años.

La información se obtuvo a partir de protocolos prospectivos de TT, bases de datos, protocolos quirúrgicos y fichas clínicas. Se calcularon los índices de gravedad de traumatismo (IGT): Injury Severity Score $^{21}$ (ISS), Revised Trauma Score Triage ${ }^{22}$ (RTST) y Trauma Injury Severity Score ${ }^{23,24}$ (TRISS). Se consideró como politraumatizado un score ISS > 16 puntos $^{25}$.

Se tabularon los datos en planilla de Microsoft Excel $^{\circledR}$ y se realizó análisis estadístico con el programa $\operatorname{SPSS} 25^{\circledR}$, con función chi-cuadrado para las variables categóricas y función ANOVA para las variables continuas entre los cuatro grupos etarios. Se consideró significativo un valor $\mathrm{p}<0,05$.

Se describen y comparan: número, sexo, clasificación, mecanismo, agente, tipo, lesiones asociadas, tratamiento, IGT, morbilidad y mortalidad de pacientes hospitalizados por TT según grupo etario.

El estudio y análisis de las hospitalizaciones por TT fue aprobado por el comité ético-científico de nuestra institución.

\section{Resultados}

En el período se hospitalizaron y fueron tratados por nuestro equipo 4.306 pacientes, de los cuales nueve se excluyeron por ser menores de 10 años, resultando un total de 4.297 pacientes para este análisis. La mayoría de ellos $(59,2 \%)$ correspondieron al grupo Adulto Joven, fue más frecuente en hombres en todos los grupos etarios y se observó un aumento significativo en la proporción de mujeres en los grupos Adulto Maduro y Adulto Mayor (Tabla 1; Figuras $1 \mathrm{~A}$ y $1 \mathrm{~B})$. 


\section{ARTíCULO ORIGINAL}

Tabla 1. Comparación de número de pacientes, edad y sexo en pacientes hospitalizados por traumatismo torácico según grupo etario

\begin{tabular}{|lcccc|}
\hline & $\begin{array}{c}\text { Adolescente } \\
(\mathbf{6 0 8})\end{array}$ & $\begin{array}{c}\text { Adulto Joven } \\
\mathbf{( 2 . 5 4 4 )}\end{array}$ & $\begin{array}{c}\text { Adulto Maduro } \\
(\mathbf{6 0 1 )}\end{array}$ & $\begin{array}{c}\text { Adulto Mayor } \\
\mathbf{( 5 4 4 )}\end{array}$ \\
Número de pacientes & $608(14,1 \%)$ & $2.544(59,2 \%)$ & $601(14,0 \%)$ & $544(12,7 \%)$ \\
Características & & & & p \\
$\quad$ Edad años (promedio) & $17,3 \pm 1,5$ & $29,2 \pm 7,0$ & $51,6 \pm 4,2$ & $71,2 \pm 8,4$ \\
Mediana & 18 & 28 & 52 & 70 \\
Sexo & & & $505(84,0 \%)$ & $366(67,3 \%)$ \\
Hombre & $571(93,9 \%)$ & $2.364(93,0 \%)$ & $96(16,0 \%)$ & $178(32,7 \%)$ \\
Mujer & $37(6,1 \%)$ & $180(7,0 \%)$ & $<0,001 *$ & $<0,001 *$ \\
\hline
\end{tabular}

*Estadísticamente significativo.

Figura 1. A: Número de pacientes. B: Sexo. C: Clasificación. D: Mecanismo del traumatismo en pacientes hospitalizados por traumatismo torácico según grupo etario. A. Joven: Adulto Joven; A. Maduro: Adulto Maduro; A. Mayor: Adulto Mayor; TT: Traumatismo torácico; Acc. tránsito: Accidente de tránsito; Acc. doméstico: Accidente doméstico.

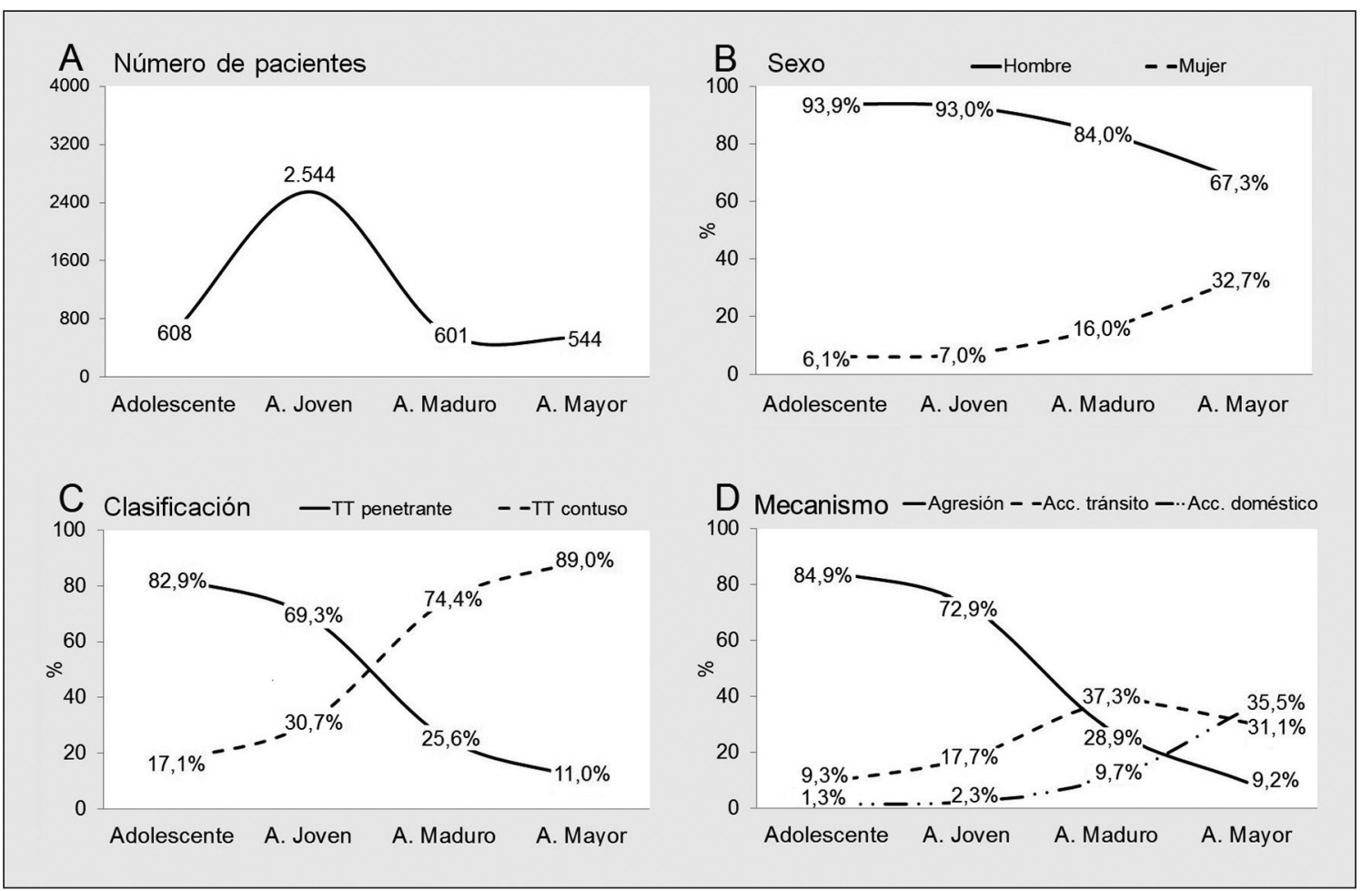

El TT penetrante predominó en los grupos jóvenes y el TT contuso fue más frecuente en los grupos de mayor edad. La agresión fue el mecanismo más prevalente de TT en los grupos Adolescente y Adulto Joven, mientras que los accidentes domésticos y de tránsito correspondieron a más de la mitad de los casos de TT del grupo Adulto Mayor (Tabla 2; Figuras 1C y 1D).

Tanto en el grupo Adolescente como en el grupo Adulto Joven el agente del TT más frecuente fue el arma blanca $(79,3 \%$ y $65,6 \%$ respectivamente), mientras que el TT por objeto contundente correspondió a un tercio de los TT en el grupo Adulto Maduro y al 55,1\% de los TT del grupo Adulto Mayor. Además, el grupo Adolescente presentó la mayor proporción de TT por arma de fuego, con diferencias estadísticamente significativas. Por otro lado, el TT aislado fue el más frecuente en los cuatro grupos etarios. En el grupo Adulto Maduro el TT con lesiones asociadas y el subgrupo de politraumatizados fueron más prevalentes que en los otros grupos con $\mathrm{p}<0,001$ (Tabla 3; Figuras 2A y 2B). 
Tabla 2. Clasificación y mecanismo del traumatismo en pacientes hospitalizados por traumatismo torácico según grupo etario

\begin{tabular}{|c|c|c|c|c|c|}
\hline & $\begin{array}{c}\text { Adolescente } \\
\text { (608) }\end{array}$ & $\begin{array}{l}\text { Adulto Joven } \\
\quad(2.544)\end{array}$ & $\begin{array}{c}\text { Adulto Maduro } \\
(601)\end{array}$ & $\begin{array}{c}\text { Adulto Mayor } \\
(544)\end{array}$ & $\mathbf{p}$ \\
\hline \multicolumn{6}{|l|}{ Clasificación } \\
\hline Penetrante & $504(82,9 \%)$ & $1.763(69,3 \%)$ & $154(25,6 \%)$ & $60(11,0 \%)$ & $<0,001 *$ \\
\hline Contuso & $104(17,1 \%)$ & $781(30,7 \%)$ & $447(74,4 \%)$ & $484(89,0 \%)$ & $<0,001 *$ \\
\hline \multicolumn{6}{|l|}{ Mecanismo } \\
\hline Agresión & $516(84,9 \%)$ & $1.854(72,9 \%)$ & $174(28,9 \%)$ & $50(9,2 \%)$ & $<0,001 *$ \\
\hline Accidente laboral & $3(0,5 \%)$ & $49(1,9 \%)$ & $47(7,8 \%)$ & $22(4,0 \%)$ & $<0,001 *$ \\
\hline Iatrogénico & $6(1,0 \%)$ & $10(0,4 \%)$ & $7(1,2 \%)$ & $18(3,3 \%)$ & $<0,001 *$ \\
\hline Autoagresión & $9(1,5 \%)$ & $19(0,7 \%)$ & $8(1,3 \%)$ & $5(0,9 \%)$ & 0,280 \\
\hline Otro mecanismo & $9(1,5 \%)$ & $104(4,1 \%)$ & $83(13,8 \%)$ & $87(16,0 \%)$ & $<0,001 *$ \\
\hline
\end{tabular}

*Estadísticamente significativo.

Tabla 3. Sitio, agente y tipo de traumatismo en pacientes hospitalizados por traumatismo torácico según grupo etario

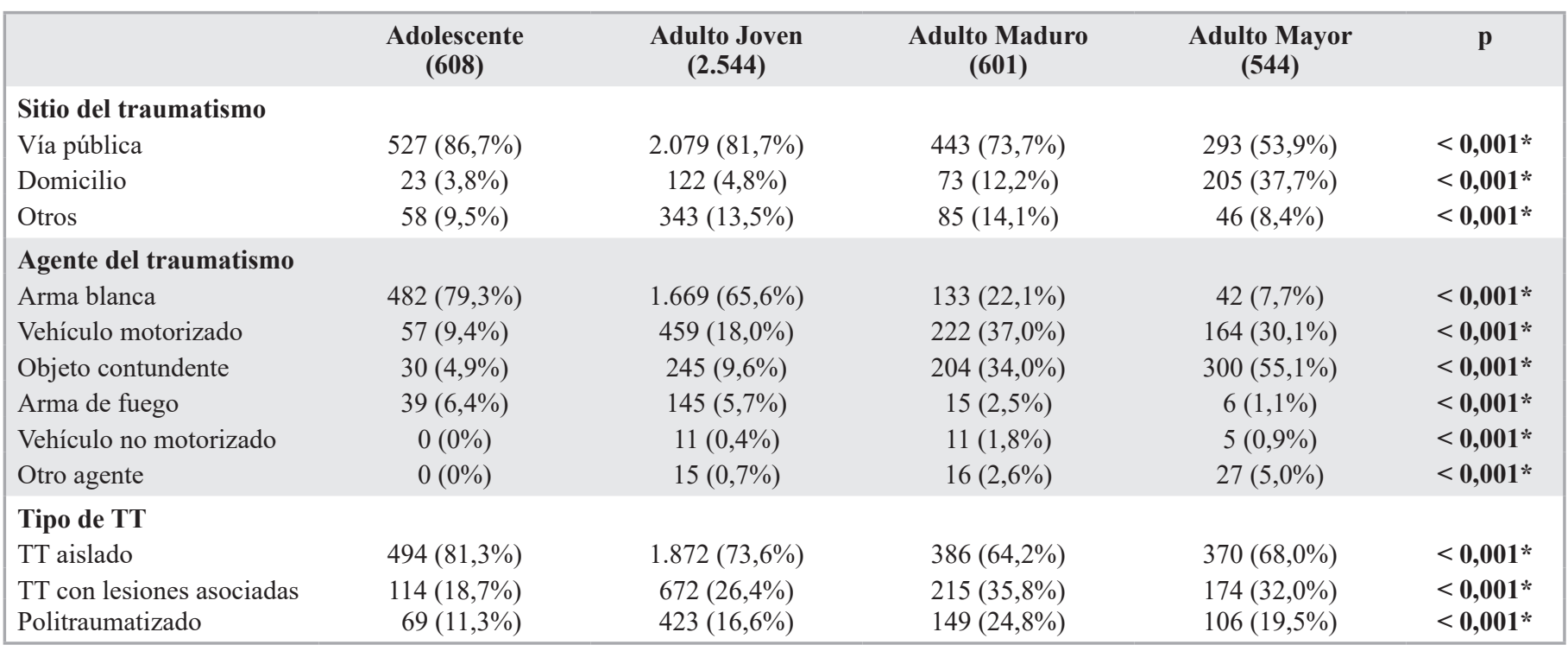

*Estadísticamente significativo; TT: Traumatismo torácico.

Las lesiones y/o hallazgos torácicos más frecuentes fueron el neumotórax y el hemotórax no masivo, siendo ambos significativamente más prevalentes en el grupo Adolescente versus los demás grupos etarios. Las fracturas costales fueron significativamente más frecuentes en el grupo Adulto Mayor alcanzando el 79,4\% de los casos. El grupo Adulto Joven presentó mayor proporción de traumatismo penetrante cardíaco y hemotórax masivo, mientras que la contusión pulmonar fue más frecuente en el grupo Adulto Maduro, todas con $\mathrm{p}<0,05$ (Tabla 4).
Las fracturas costales y sus complicaciones, así como las lesiones extratorácicas más frecuentemente encontradas en TT se detallan en las Tablas 5 y 6 respectivamente. Aunque las fracturas costales fueron más frecuentes en el Adulto Mayor, las complicaciones de estas y el tórax volante fueron proporcionalmente mayores en el grupo Adulto Maduro con $\mathrm{p}<0,001$. Así mismo, las lesiones craneoencefálicas y esqueléticas fueron más frecuentes en el grupo Adulto Maduro comparado con los otros grupos etarios, con diferencias estadísticamente significativas. 


\section{ARTÍ́CULO ORIGINAL}

Figura 2. A: Agente. B: Tipo de TT. C: Tratamiento. D: Tratamiento invasivo en pacientes hospitalizados por traumatismo torácico según grupo etario. TT: Traumatismo torácico; Veh. Motorizado: Vehículo Motorizado; Ob. Contundente: Objeto Contundente; A. Joven: Adulto Joven; A. Maduro: Adulto Maduro; A. Mayor: Adulto Mayor.

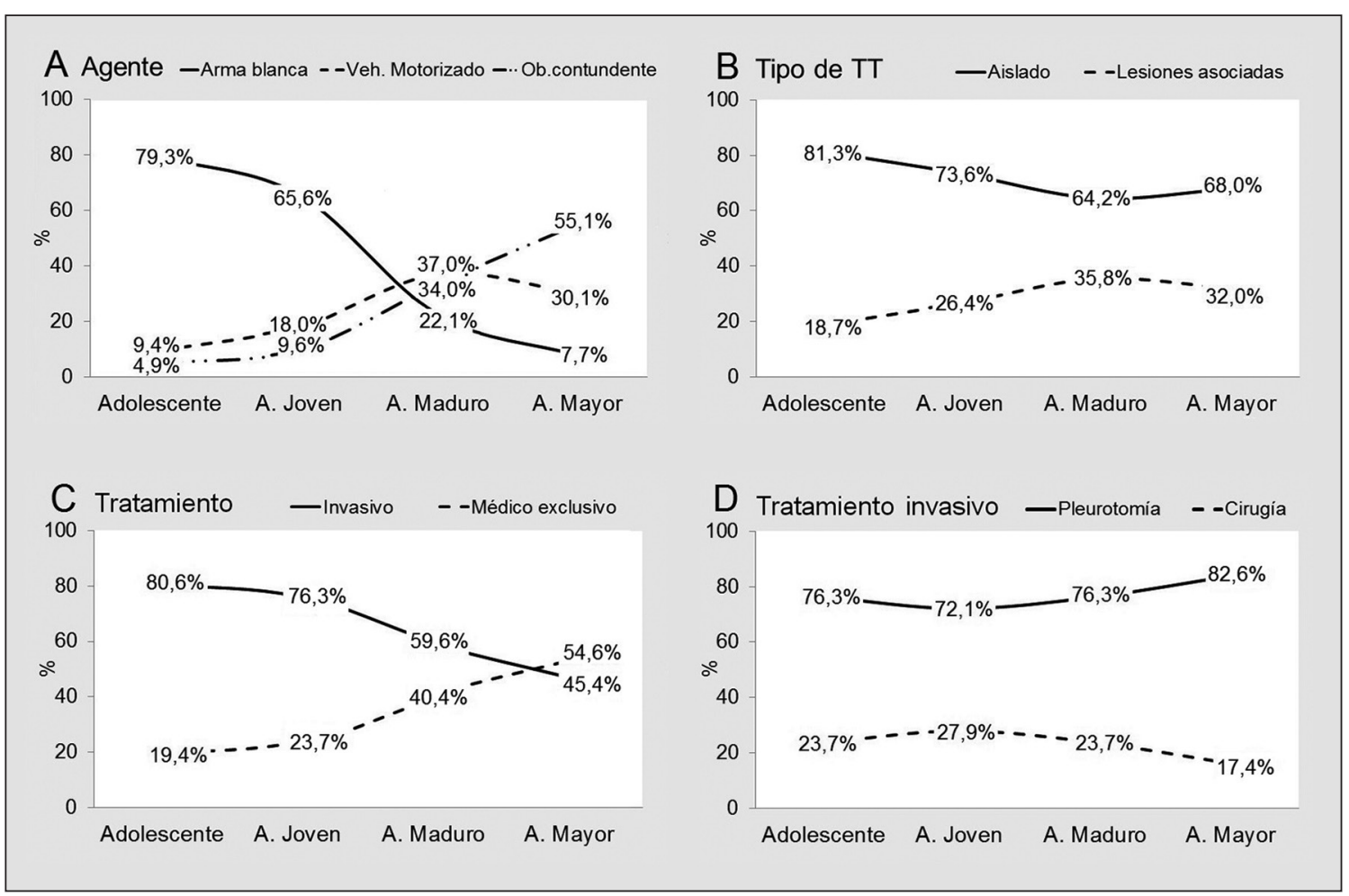

Tabla 4. Hallazgos y/o lesiones torácicas en pacientes hospitalizados por traumatismo torácico según grupo etario

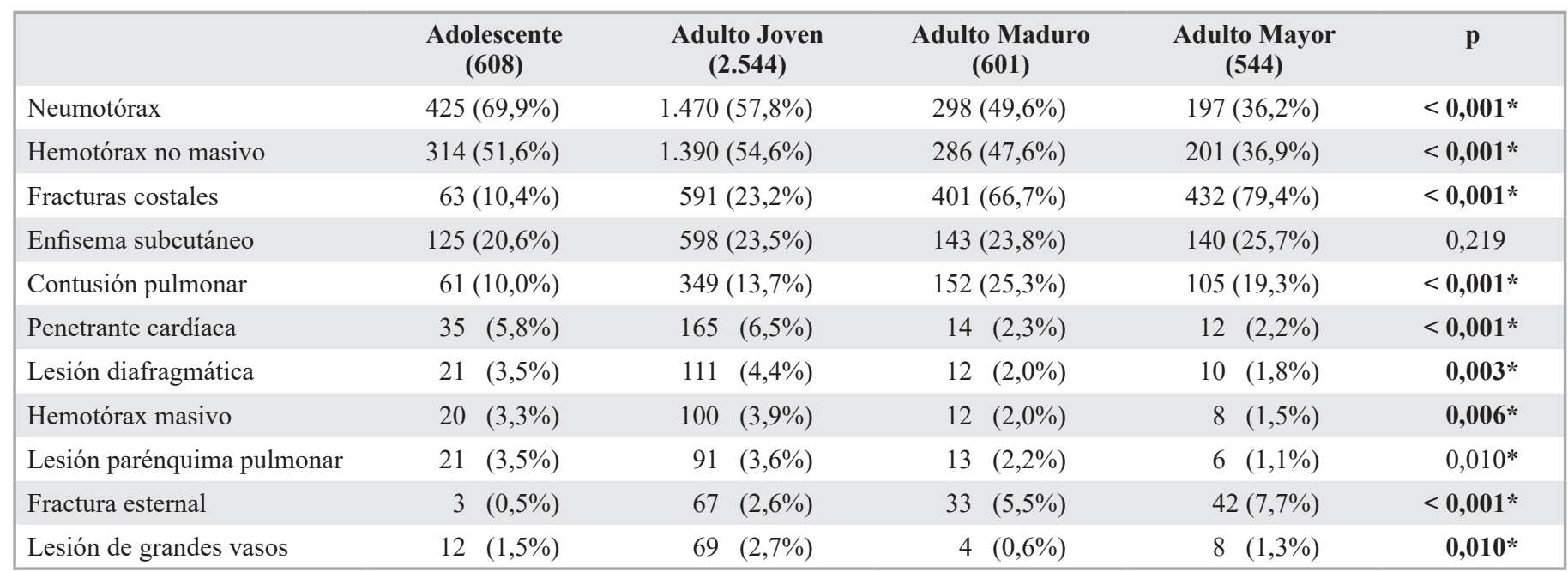

*Estadísticamente significativo.

Respecto al tratamiento definitivo, el tratamiento invasivo fue el más realizado en todos los grupos etarios excepto en el grupo Adulto Mayor, donde se efectuó tratamiento médico exclusivo en un $54,6 \%$ de los casos. La pleurotomía fue el tratamiento de elección en tres cuartas partes de los pacientes con tratamiento invasivo, mientras que el menor número de cirugías se registró en el grupo Adulto Mayor, con $\mathrm{p}<0,001$ (Tabla 7, Figuras 2C y 2D).

Se observaron diferencias significativas en la 
Tabla 5. Fracturas costales y sus complicaciones en relación al total de pacientes hospitalizados por traumatismo torácico según grupo etario

\begin{tabular}{|c|c|c|c|c|c|}
\hline & $\begin{array}{c}\text { Adolescente } \\
\text { (608) }\end{array}$ & $\begin{array}{l}\text { Adulto Joven } \\
\quad(2.544)\end{array}$ & $\begin{array}{c}\text { Adulto Maduro } \\
(601)\end{array}$ & $\begin{array}{c}\text { Adulto Mayor } \\
\text { (544) }\end{array}$ & $\mathbf{p}$ \\
\hline \multicolumn{6}{|l|}{ Fracturas costales } \\
\hline 1 a 3 costillas & $36(5,9 \%)$ & $309(12,2 \%)$ & $171(28,5 \%)$ & $214(39,3 \%)$ & $<0,001 *$ \\
\hline 4 a 6 costillas & $21(3,5 \%)$ & $193(7,6 \%)$ & $157(26,0 \%)$ & $152(27,9 \%)$ & $<0,001 *$ \\
\hline$>6$ costillas & $6(1,0 \%)$ & $89(3,5 \%)$ & $73(12,2 \%)$ & $66(12,1 \%)$ & $<0,001 *$ \\
\hline Tórax volante & $10(1,6 \%)$ & $81(3,2 \%)$ & $67(11,2 \%)$ & $51(9,4 \%)$ & $<0,001^{*}$ \\
\hline FC con complicación intratorácica & $56(9,2 \%)$ & $489(19,2 \%)$ & $309(51,4 \%)$ & $276(50,7 \%)$ & $<0,001 *$ \\
\hline FC con complicación extratorácica & $14(2,3 \%)$ & $91(3,6 \%)$ & $45(7,5 \%)$ & $25(4,6 \%)$ & $<0,001 *$ \\
\hline
\end{tabular}

*Estadísticamente significativo; FC: Fracturas costales.

Tabla 6. Lesiones extratorácicas en pacientes hospitalizados por traumatismo torácico según grupo etario

\begin{tabular}{|c|c|c|c|c|c|}
\hline & $\begin{array}{l}\text { Adolescente } \\
\qquad(608)\end{array}$ & $\begin{array}{c}\text { Adulto Joven } \\
\qquad(2.544)\end{array}$ & $\begin{array}{l}\text { Adulto Maduro } \\
(601)\end{array}$ & $\begin{array}{c}\text { Adulto Mayor } \\
\text { (544) }\end{array}$ & p \\
\hline Craneoencefálicas & $35(5,8 \%)$ & $258(10,1 \%)$ & $101(16,8 \%)$ & $83(15,3 \%)$ & $<0,001 *$ \\
\hline Extremidades & $38(6,3 \%)$ & $191 \quad(7,5 \%)$ & $78(13,0 \%)$ & $64(11,8 \%)$ & $<0,001 *$ \\
\hline Fracturas de pelvis & $9(1,5 \%)$ & $70 \quad(2,8 \%)$ & $37 \quad(6,2 \%)$ & $28 \quad(5,1 \%)$ & $<0,001 *$ \\
\hline Faciales & $13(2,1 \%)$ & $60 \quad(2,4 \%)$ & $26 \quad(4,3 \%)$ & $25 \quad(4,6 \%)$ & $0,003 *$ \\
\hline
\end{tabular}

*Estadísticamente significativo.

Tabla 7. Tratamiento definitivo realizado en pacientes hospitalizados por traumatismo torácico según grupo etario

\begin{tabular}{|c|c|c|c|c|c|}
\hline & $\begin{array}{l}\text { Adolescente } \\
\quad(608)\end{array}$ & $\begin{array}{c}\text { Adulto Joven } \\
\quad(2.544)\end{array}$ & $\begin{array}{c}\text { Adulto Maduro } \\
\text { (601) }\end{array}$ & $\begin{array}{c}\text { Adulto Mayor } \\
(544)\end{array}$ & p \\
\hline Tratamiento invasivo & $490(80,6 \%)$ & $1.942(76,3 \%)$ & $358(59,6 \%)$ & $247(45,4 \%)$ & $<0,001 *$ \\
\hline Cirugía & $116(19,1 \%)$ & $541(21,2 \%)$ & $85(14,2 \%)$ & $43 \quad(7,9 \%)$ & $<0,001 *$ \\
\hline Médico & $118(19,4 \%)$ & $602(23,7 \%)$ & $243(40,4 \%)$ & $297(54,6 \%)$ & $<0,001 *$ \\
\hline
\end{tabular}

*Estadísticamente significativo.

morbilidad, mortalidad y estadía hospitalaria entre los distintos grupos etarios. El grupo Adulto Maduro presentó la mayor morbilidad con un $20,8 \%$, en tanto que en el grupo Adulto Mayor se evidenció una mortalidad general y por TT aislado significativamente mayor comparado con los otros grupos etarios. Por otro lado, aunque no fue estadísticamente significativo, la mortalidad del politraumatismo en el grupo Adulto Mayor (15,1\%) fue más del doble con respecto al grupo Adolescente (7,3\%) (Tabla 8; Figuras 3A y 3B).

En relación a los promedios de IGT, el ISS fue significativamente mayor en los grupos de edad intermedia, no se observaron diferencias en el RTS-T al momento del ingreso y el TRISS fue significativamente superior en el grupo Adulto Mayor con $\mathrm{p}<0,001$. La mortalidad observada fue menor a la esperada en los cuatro grupos etarios (Tabla 9). 


\section{ARTíCULO ORIGINAL}

Tabla 8. Morbilidad, mortalidad y estadía hospitalaria en pacientes hospitalizados por traumatismo torácico según grupo etario

\begin{tabular}{|c|c|c|c|c|c|}
\hline & $\begin{array}{c}\text { Adolescente } \\
\text { (608) }\end{array}$ & $\begin{array}{l}\text { Adulto Joven } \\
\qquad(2.544)\end{array}$ & $\begin{array}{c}\text { Adulto Maduro } \\
\qquad(601)\end{array}$ & $\begin{array}{c}\text { Adulto Mayor } \\
\text { (544) }\end{array}$ & $\mathbf{p}$ \\
\hline Morbilidad & $74(12,2 \%)$ & $412(16,2 \%)$ & $125(20,8 \%)$ & $80(14,7 \%)$ & $0,001 *$ \\
\hline TT aislado & $4 / 494 \quad(0,8 \%)$ & $23 / 1.872 \quad(1,2 \%)$ & $1 / 386 \quad(0,3 \%)$ & $11 / 370 \quad(3,0 \%)$ & $0,005 *$ \\
\hline TT con lesiones asociadas & $6 / 114 \quad(4,2 \%)$ & $40 / 672 \quad(6,0 \%)$ & $19 / 215 \quad(8,9 \%)$ & $16 / 174 \quad(9,2 \%)$ & 0,300 \\
\hline Politraumatizado & $5 / 69 \quad(7,3 \%)$ & $37 / 423(8,5 \%)$ & $18 / 149(12,1 \%)$ & $16 / 106(15,1 \%)$ & $* *$ \\
\hline Promedio & $6,5 \pm 7,0$ & $7,5 \pm 8,6$ & $9,8 \pm 9,9$ & $9,1 \pm 8,8$ & $<0,001 *$ \\
\hline Mediana & 4 & 5 & 7 & 6 & \\
\hline Rango & $1-60$ & $1-150$ & $1-91$ & $1-83$ & \\
\hline
\end{tabular}

*Estadísticamente significativo; TT: Traumatismo torácico. **En el grupo específico de pacientes politraumatizados, la mortalidad del grupo etario Adulto Mayor fue superior a los grupos Adulto Maduro y Adulto Joven; y más del doble con respecto al grupo Adolescente.

Tabla 9. Índices de gravedad del traumatismo en pacientes hospitalizados por traumatismo torácico según grupo etario

\begin{tabular}{|c|c|c|c|c|c|}
\hline & $\begin{array}{l}\text { Adolescente } \\
\qquad(608)\end{array}$ & $\begin{array}{l}\text { Adulto Joven } \\
\quad(2.544)\end{array}$ & $\begin{array}{c}\text { Adulto Maduro } \\
\text { (601) }\end{array}$ & $\begin{array}{l}\text { Adulto Mayor } \\
\qquad(544)\end{array}$ & p \\
\hline $\begin{array}{l}\text { Injury Severity Score (ISS) } \\
\text { Promedio }\end{array}$ & $11,9 \pm 7,8$ & $13,1 \pm 9,7$ & $13,6 \pm 9,7$ & $12,2 \pm 9,6$ & $0,002 *$ \\
\hline $\begin{array}{l}\text { Revised Trauma Score T }(R T S-T) \\
\text { Promedio }\end{array}$ & $11,6 \pm 1,4$ & $11,5 \pm 1,6$ & $11,4 \pm 1,7$ & $11,6 \pm 1,3$ & 0,173 \\
\hline $\begin{array}{l}\text { Trauma Injury Severity Score (TRISS) } \\
\text { Promedio (\% de mortalidad esperada) }\end{array}$ & 4,1 & 4,8 & 7,1 & 8,4 & $<0,001 *$ \\
\hline Mortalidad observada & $1,6 \%$ & $2,5 \%$ & $3,3 \%$ & $5,0 \%$ & $0,003 *$ \\
\hline
\end{tabular}

*Estadísticamente significativo.

Figura 3. A: Mortalidad esperada y observada. B: Mortalidad observada según tipo de traumatismo en pacientes hospitalizados por traumatismo torácico y grupo etario. TRISS: Trauma Injury Severity Score; A. Joven: Adulto Joven; A. Maduro: Adulto Maduro; A. Mayor: Adulto Mayor.

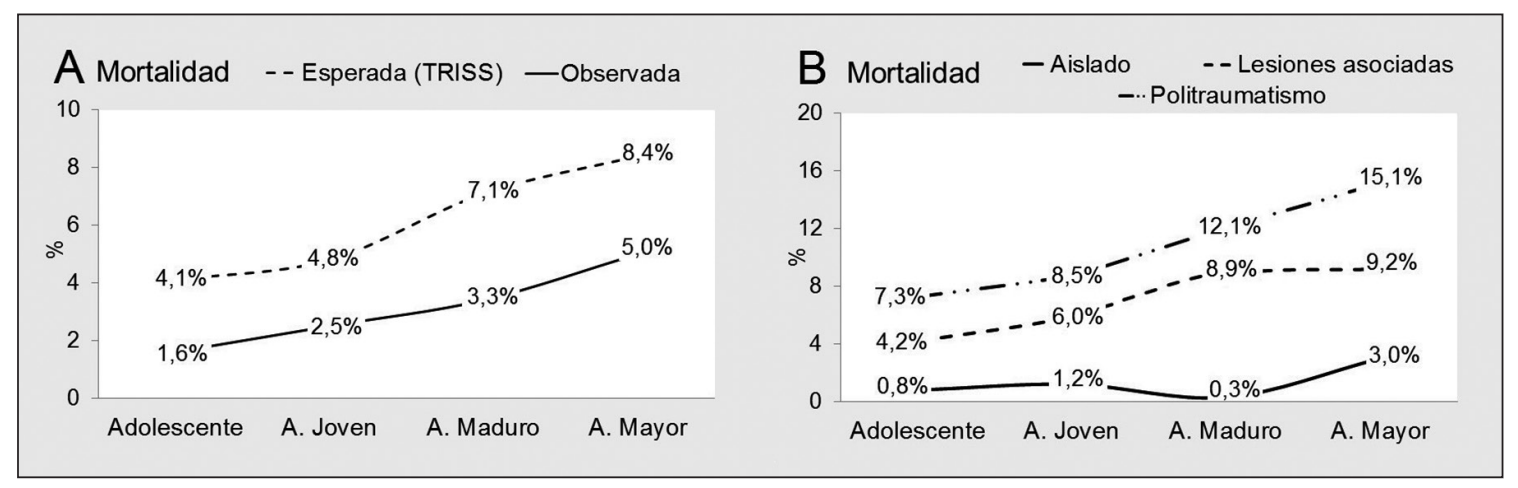

\section{Discusión}

Existen diferencias estadísticamente significativas en las características clínicas, IGT y mortalidad del TT entre distintos grupos etarios. Estas diferencias fueron especialmente evidentes en los grupos de edad extremos de esta serie. De este modo, en nuestro medio, el TT en el grupo Adolescente se caracterizó por ser en su mayoría penetrante y aislado, debido a agresión por arma blanca y arma de fuego, que requirió tratamiento invasivo y tuvo baja morbimortalidad versus el grupo Adulto Mayor donde fue más frecuente el contuso, asociado a fracturas costales, con mayor proporción de tratamiento 
médico y con peores IGT, morbilidad y mortalidad, especialmente en el subgrupo de politraumatizados.

Desde el punto de vista epidemiológico, nuestra serie presentó mayoritariamente hospitalizaciones por TT en el grupo Adulto Joven, lo que es concordante con series donde existe alta frecuencia de agresiones $^{9,26}$. Destacamos que la gran mayoría de las agresiones por arma de fuego ocurrieron en los dos grupos etarios más jóvenes, observándose una significativa mayor proporción en el grupo Adolescente. Esto, asociado a un aumento progresivo de este tipo de traumatismo en nuestro medio, es consistente con lo comunicado en series internacionales donde los traumatismos por arma de fuego representan una importante causa de morbilidad y mortalidad en este grupo etario ${ }^{27,28}$.

La mayoría de las características clínicas del TT en el grupo Adulto Joven están en un rango intermedio entre los grupos Adolescente y Adulto Maduro, sin embargo, el análisis evidenció que este grupo etario posee características particulares; entre ellas, una mayor y significativa proporción de traumatismo penetrante cardíaco y hemotórax masivo. Ambas corresponden a urgencias quirúrgicas con características clínicas propias que determinan una mayor gravedad del TT y se asocian a mayor mortalidad $^{29,30}$. Esto podría explicar la mayor mortalidad observada del subgrupo de TT aislado en el grupo Adulto Joven versus los grupos Adolescente o Adulto Maduro.

El grupo Adulto Maduro representa una población laboralmente activa que es susceptible a accidentes de tránsito y laborales ${ }^{31}$. En nuestra serie, se encontraron las frecuencias más altas de politraumatizados, así como de tórax volante y de fracturas costales con complicación intra y extratorácica en este grupo etario. Esto podría deberse a que se encuentran más expuestos a los TT de alta energía, como los accidentes de tránsito; contrario a lo que ocurre en el grupo Adulto Mayor donde predominaron los TT de baja energía (caídas) o a los grupos más jóvenes, donde fueron más frecuentes los traumatismos por violencia civil. Además, estos hallazgos concuerdan con lo informado a nivel nacional, en que el grupo Adulto Maduro concentra la mayor tasa ajustada por edad de defunciones debido a accidentes de tránsito (12,8 defunciones por 100.000 habitantes) comparado con los demás grupos etarios $^{32}$.

Más aún, la significativa mayor proporción de lesiones extratorácicas (craneoencefálicas, extremidades y fractura de pelvis) encontradas en el grupo Adulto Maduro tributó directamente en un mayor puntaje ISS, teniendo este grupo etario lesiones significativamente más graves que el grupo Adulto Mayor, sin que ello significase una mayor mortalidad observada. Este hallazgo reafirma el hecho de que la edad es un factor independiente de mortalidad en $\mathrm{TT}^{33,34}$.

Sin embargo, la evidencia disponible le atribuye mayor preponderancia a la edad como factor de riesgo de mortalidad por TT contuso por sobre el TT penetrante, debido a que la mortalidad en este último está supeditada a otros factores como el traumatismo penetrante cardíaco, el hemotórax masivo o las lesiones de grandes vasos, los cuales pueden distribuirse de forma más homogénea entre distintos grupos etarios de diferentes series. En el TT contuso, por otro lado, la edad se asocia a mayor mortalidad a expensas del deterioro fisiológico propio del envejecimiento, las comorbilidades cardiopulmonares, la fragilidad y la mayor frecuencia de fracturas costales múltiples, entre otros ${ }^{9,14,35-39}$.

El grupo Adulto Mayor representa una población cada vez más frecuente en nuestro medio. Las características del TT en este grupo etario han sido descritas y difieren de la población general ${ }^{14,18,40,41}$. Estas diferencias pueden explicarse fisiopatológicamente, en parte, por los cambios que ocurren en el tórax óseo durante el envejecimiento.

Normalmente la densidad mineral ósea aumenta proporcionalmente hasta los 30 años, se mantiene estable entre los 30 y 40 años y luego disminuye aceleradamente hasta la vejez; etapa donde se ha demostrado que la dureza de las costillas es hasta tres veces menor que en personas jóvenes ${ }^{11}$. En este proceso los principales factores que alteran las propiedades mecánicas del tórax son el aumento de la porosidad ósea (disminución de la densidad) y el cambio en el contenido mineral óseo (desmineralización), los que favorecen directamente la aparición de fracturas costales ante traumatismos de baja energía como las caídas a nivel ${ }^{11,12}$. Publicaciones recientes recomiendan un enfoque principalmente conservador en el tratamiento del TT en adultos mayores, con énfasis en un adecuado alivio del dolor por sobre otros procedimientos invasivos ${ }^{42}$.

Una de las limitaciones de nuestro análisis fue que se emplearon cuatro grandes grupos etarios con intervalos de edades amplios, lo que puede enmascarar ciertos patrones de distribución clínica como lo son la presentación trimodal de la contusión pulmonar y/o de la fractura esternal; modelos que han sido reproducidos en series internacionales utilizando múltiples grupos etarios con intervalos de edad reducidos ${ }^{43,44}$. Por otro lado, el grupo Adolescente podría estar subrepresentado debido a que existen casos entre 10 y 15 años que no necesariamente 
son evaluados y tratados por nuestro equipo. Pese a esto, una de las fortalezas de esta serie es el número de casos, que aseguró la representatividad de cada grupo etario; así como una adecuada proporción entre TT contusos y penetrantes, lo que permitió disminuir sesgos de interpretación, haciendo esta serie comparable con estudios internacionales.

En conclusión, existen diferencias estadísticamente significativas en las características del TT entre diferentes grupos etarios. Es más frecuente en hombres jóvenes y presenta peores IGT, morbilidad y mortalidad en pacientes ancianos, especialmente en el subgrupo de politraumatizados. Finalmente, la edad es uno de los principales factores que deter- mina el pronóstico de los pacientes hospitalizados por TT.

\section{Responsabilidades éticas}

Protección de personas y animales. Los autores declaran que para esta investigación no se han realizado experimentos en seres humanos ni en animales.

Confidencialidad de los datos. Los autores declaran que en este artículo no aparecen datos de pacientes.

Conflictos de interés: no hay.

\section{Bibliografía}

1. Murray CJ, López AD. Mortality by cause for eight regions of the world: Global Burden of Disease Study. Lancet 1997;349:1269-81.

2. Demirhan R, Onan B, Oz K, Halezeroglu S. Comprehensive analysis of 4205 patients with chest trauma: a 10-year experience. Interact Cardiovasc Thorac Surg. 2009;9:450-3.

3. El-Menyar A, Latifi R, AbdulRahman H, Zarour A, Tuma M, Parchani A, et al. Age and traumatic chest injury: a 3-year observational study. Eur J Trauma Emerg Surg. 2013;39:397-403.

4. Al-Koudmani I, Darwish B, Al-Kateb $\mathrm{K}$, Taifour Y. Chest trauma experience over eleven-year period at Al-Mouassat University Teaching Hospital-Damascus: a retrospective review of 888 cases. J Cardiothorac Surg. 2012;7:35.

5. Departamento de estadística e información en salud. Defunciones y mortalidad por causas. Disponible en: www.deis.cl/ estadísticas-mortalidad/.

6. Bächler R, Icaza G, Soto A, Núñez L, Orellana C, Monsalve R, et al. Epidemiología de las muertes prematuras en Chile en la década 2001-2010. Rev Med Chile 2017;145:319-26.

7. Departamento de estadística e información en salud. Egresos hospitalarios 2012-2018 según edad y causa. Disponible en: www. deis.minsal.cl.

8. Wanek S, Mayberry JC. Blunt thoracic trauma: flail chest, pulmonary contusion, and blast injury. Crit Care Clin. 2004;20:71-81.
9. Peterson RJ, Tepas JJ, Edwards FH, Kissoon N, Pieper P, Ceithaml EL. Pediatric and adult thoracic trauma: age-related impact on presentation and outcome. Ann Thorac Surg. 1994;58:14-8.

10. Mollberg NM, Tabachnick D, Lin FJ, Merlotti GJ, Varghese TK, Arensman RM, et al. Age-associated impact on presentation and outcome for penetrating thoracic trauma in the adult and pediatric patient populations. J Trauma Acute Care Surg. 2014;76:273-8.

11. Zhou Q, Rouhana SW, Melvin JW. Age effects on thoracic injury tolerance. SAE Tech Pap. 1996;962421.

12. Émond $\mathrm{M}$, Sirois MJ, Guimont $\mathrm{C}$, Chauny JM, Daoust R, Bergeron E, et al. Funtional impact of a minor thoracic injury. Ann Surg. 2015;262:1115-22.

13. González R, Riquelme A, Fuentes A, Saldías R, Reyes R, Seguel E, et al. Traumatismo torácico: caracterización de hospitalizaciones durante tres décadas. Rev Med Chile 2018;146:196-205.

14. González R, Fuentes A, Riquelme A, Reyes R, Seguel E, Stockins A, et al. Traumatismo torácico en el adulto mayor. Rev Cir. 2020;72:224-30.

15. Ceran S, Sunam GS, Aribas OK, Gormus $\mathrm{N}$, Solak H. Chest trauma in children. Eur J Cardiothorac Surg. 2002;21:57-9.

16. Talving P, Demetriades D. Cardiac trauma during teenage years. Pediatr Clin N Am. 2014;61:111-30.

17. Aschkenasy M, Rothenhaus T. Trauma and falls in the elderly. Emerg Med Clin N Am. 2006;24:413-32.

18. Inci I, Ozçelik C, Nizam O, Eren N. Thoracic trauma in the elderly. Eur J
Emerg Med. 1998;5:445-50.

19. Organización Mundial de la Salud. Competencias básicas en materia de salud y desarrollo de los adolescentes para proveedores de atención primaria [internet] 2015. [citado 29 abril de 2020]. Disponible en: www.who.int/ maternal_child_adolescent/publications/ core_competencies_adolescents/es/

20. Informe mundial sobre el envejecimiento y salud. En: Organización Mundial de la Salud. 2015. WHO/FWC/ALC/15.01.

21. Baker SP, O’Neill B, Haddon W, Long W. The Injury Severity Score: a method for describing patients with multiple injuries and evaluating emergency care. J Trauma 1974;14:187-96.

22. Champion H, Sacco W, Copes W, Gann D, Gennarelli T, Flanagan M. A revision of the Trauma Score. J Trauma 1989;29:6239.

23. Boyd C, Tolson M, Copes W. Evaluating trauma care: the TRISS method. Trauma Score and Injury Severity Score. J Trauma 1987;27:370-8.

24. Freixinet J, Beltrán J, Rodríguez P, Juliá G, Hussein M, Gil R, et al. Indicators of Severity in Chest Trauma. Arch Bronconeumol. 2008:44:257-62.

25. Mica L, Rufibach K, Keel M, Trentz O. The risk of early mortality of polytrauma patients associated to ISS, NISS, APACHE II values and prothrombin time. J Trauma Manag Outcomes 2013;7:6.

26. González R, Riquelme A, Toloza C, Reyes R, Seguel E, Stockins A, et al. Traumatismo torácico por arma blanca. Rev Cir. 2020;72:137-43.

27. González R, Riquelme A, Ávalos Á, 
Reyes R, Seguel E, Stockins A, et al. Traumatismo torácico por arma de fuego. Rev Cir. 2020;72:[In press].

28. The Global Burden of Disease 2016 Injury Collaborators. Global mortality from firearms, 1990-2016. JAMA 2018;320:792-814.

29. González R, Riquelme A, Fuentes A, Canales J, Seguel E, Stockins A, et al. Traumatismo penetrante cardíaco: caracterización, resultados inmediatos y variables asociadas a morbilidad y mortalidad en pacientes operados. Rev Cir. 2019;71:245-52.

30. González R, Riquelme A, Toloza C, Reyes R, Seguel E, Stockins A, et al. Hemotórax masivo por traumatismo torácico en pacientes tratados quirúrgicamente. Rev Cir. 2020;72: [In press].

31. Stitzel JD, Kilgo PD, Weaver AA, Martin RS, Loftis KL, Meredith JW, et al. Age thresholds for increased mortality of predominant crash induced thoracic injuries. Ann Adv Automov Med. 2010;54:41-50.

32. Departamento de Estadística e Información en salud. Serie defunciones por accidentes de transporte. Disponible en: www.deis.cl/estadísticas-mortalidad/.
33. Heinrich D, Holzmann C, Wagner A, Fischer A, Pfeifer R, Graw M, et al. What are the differences in injury patterns of young and elderly traffic accident fatalities considering death on scene and death in hospital? Int J Legal Med. 2017;131:102337.

34. Campbell-Furtick M, Moore BJ, Overton TL, Phillips JL, Simon KJ, Gandhi RG, et al. Post-trauma mortality at age 60: a cutoff for defining elderly? Am J Surg. 2016;212:781-5

35. Hanafi M, Al-Sarraf N, Sharaf H, Abdelaziz A. Pattern and presentation of blunt chest trauma among different age groups. Asian Cardiovasc Thorac Ann. 2011; 19: 48-51.

36. González R, Riquelme A, Toloza C, Reyes R, Seguel E, Stockins A, et al Traumatismo torácico contuso. Rev Chil Enferm Respir. 2019;35:96-103.

37. Holcomb JB, McMullin NR, Kozar RA, Lygas MH, Moore FA. Morbidity from rib fractures increases after age 45. J Am Coll Surg. 2003;196:549-55.

38. Battle CE, Hutchings H, Evans PA. Risk factors that predict mortality in patients with blunt chest wall trauma: A systematic review and meta-analysis. Injury
2012;43:8-17.

39. Kessel B, Dagan J, Swaid F, Ashkenazi I, Olsha O, Peleg K, et al. Rib fractures: comparison of associated injuries between pediatric and adult population. Am J Surg. 2014;208:831-4.

40. Shorr RM, Rodríguez A, Indeck MC, Crittenden MD, Hartunian S, Cowley RA. Blunt chest trauma in the elderly. J Trauma 1989;29:234-7.

41. Bader A, Rahman U, Morris M, McCormack JE, Huang EC, Zawin $\mathrm{M}$, et al. Pulmonary contusions in the elderly after blunt trauma: incidence and outcomes. J Surg Res. 2018;230:110-6.

42. Vana PG, Mayberry J, Luchette FA. Management and complications of rib fractures in older adults. Curr Geri Rep. 2016;5:25-30.

43. Schmitt S, Krinner S, Langerbach A, Henning FF, Schulz-Drost S. Analysis on the age distribution of sternum fractures. Thorac Cardiovasc Surg. 2018;66:670-7.

44. Finkbeiner R, Krinner S, Langerbach A, Besendörfer M, Schulz-Drost S. Age distribution and concomitant injuries in pulmonary contusion: an analysis based on routine data. Thorac Cardiovasc Surg. 2018;66:678-85. 